\title{
No contribution of GSTM1 and GSTT1 null genotypes to the risk of neutropenia due to benzene exposure in Southeastern Brazil
}

\author{
Carmen Silvia Passos Lima, Gustavo Jacob Lourenço, Irene Lorand-Metze, Helvia Nascimento, \\ Sara Teresinha Ollala Saad and Fernando Ferreira Costa \\ Departamento de Clínica Médica, Faculdade de Ciências Médicas, Universidade Estadual de Campinas, \\ Campinas, SP, Brazil.
}

\begin{abstract}
Exposure to benzene has been associated with haematological diseases such as neutropenia (NEB) and acute myeloid leukaemia (AML). We tested whether the null genotypes of the GSTM1 and GSTT1 genes, involved in benzene inactivation, altered the risk for NEB in southeastern Brazil. Genomic DNA from 55 NEB patients and 330 controls was analysed by multiplex-polymerase chain reaction. The frequency of the GSTM1, GSTT1 and combined null genotypes was similar in patients and controls (GSTM1, 27.3\% vs. 38.8\%, p = 0.16; GSTT1, 25.5\% vs. $19.7 \%$, $\mathrm{p}=0.24$; GSTM1/GSTT1, $12.7 \%$ vs. $6.7 \%, \mathrm{p}=0.26$; respectively). The distribution of genotype classes in NEB patients was similar to normal controls, suggesting that GSTM1 and GSTT1 null genotypes make no specific contribution to the risk of NEB. As the GSTM1 and GSTT1 null genotypes were previously associated with increased risk for $\mathrm{AML}$ in Brazil and elsewhere, we hypothesise that different thresholds of chemical exposure relative to distinct GSTM1 and GSTT1 genotypes may determine whether AML or NEB manifests in benzene exposed individuals from southeastern Brazil. Although indicative, our results still require support by prospective and large scale epidemiological studies, with rigorous assessment of daily chemical exposures and control of the possible contribution of other polymorphic genes involved in benzene metabolism.
\end{abstract}

Key words: neutropenia, glutathione S-transferase, GSTM1, GSTT1.

Received: November 27, 2008; Accepted: May 13, 2009.

The past decade has seen an increasing interest in associations of haematological diseases and occupational exposure to chemical agents. In this context, neutropenia due to exposure to benzene (NEB) has generally been characterised by slight to moderate decrease in neutrophil count (Lorand et al., 1984; Ruiz et al., 1994; Queiroz et al., 1997; Augusto et al., 1999). In contrast, a marked reduction in erythrocyte, neutrophil and platelet counts has been seen in acute myeloid leukaemia (AML), a more severe form of occupational disease (Vaughan et al., 2005). Glutathione Stransferases (GST) modulate the effects of exposure to chemical agents linked to NEB and AML (Hayes et al., 2005). The GSTM1 and GSTT1 genes have null variant genotypes, which have been associated with AML susceptibility (Ye and Song, 2005).

Occupation-related diseases have been described as a serious health problem in southeastern Brazil (Lorand et al., 1984; Ruiz et al., 1994; Queiroz et al., 1997; Augusto et

Send correspondence to Carmen Silvia Passos Lima. Clinical Oncology Service, Department of Internal Medicine, Faculty of Medical Sciences, Universidade Estadual de Campinas, Cidade Universitária "Zeferino Vaz", Rua Alexander Fleming 181, 13083-970 Distrito de Barão Geraldo, Campinas, SP, Brazil. E-mail: carmenl@fcm.unicamp.br. al., 1999), and associations of the GST null genotypes and increased risks for AML were previously reported by us in individuals from this area of the country (Arruda et al., 2001). Moreover, to the best of our knowledge, no studies regarding the associations of the GSTM1 and GSTT1 genotypes and NEB risk have been reported. For these reasons, the identification of genotypes of the GSTMI and GST1 genes in NEB patients from southeastern Brazil was considered necessary in order to investigate their influence, if any, as a risk factor for NEB.

We analysed 55 consecutive NEB patients (34 men, 21 women; 29 Caucasians, 26 African-Brazilians; mean age $35 \pm 13$ years) seen at the hospital of the State University of Campinas, from January 2002 to December 2005. Neutropenia was defined as a neutrophil count below $1.8 \times 10^{9} / \mathrm{L}$ for Caucasians and $1.5 \times 10^{9} / \mathrm{L}$ for African-Brazilians (Watts, 1999; Dale, 2001). NEB patients presented a consistent history of daily benzene occupational exposure (they were painters, mechanics, shoemakers, or workers of construction companies, petrochemical industry and petrol stations) for a period of over 12 months. Hypocellularity of the granulocytic lineage in bone marrow and exclusion of other causes of neutropenia, such as infectious, autoimmune, haematological, thyroid and nutritional deficiency or 
drug-related neutropenia, as previously recommended by our group (Lima et al., 2006), were also required for NEB diagnosis. The control group consisted of 330 blood donors (247 men, 83 women; 174 Caucasians, 156 African-Brazilians; mean age $51 \pm 3$ years) without a consistent history of benzene exposure. They were recruited from the same university hospital in order to provide a representative group of the general population that seeks medical assistance in the region. All procedures were carried out according to the principles of the institutional guidelines and all patients and controls provided written informed consent.

Genomic DNA from peripheral blood of patients and controls was analysed by multiplex-polymerase chain reaction for identification of GST genotypes (Arruda et al., 2001). The GST genotypes were analysed after electrophoresis on $2.0 \%$ agarose gels (Figure 1 ), using the $\beta$-globin gene as internal control.

Differences between groups were analysed by means of chi-squared or Fishers exact tests. For analysing the associations with NEB, univariate and multivariate analyses were used throughout, in order to obtain odds ratio (OR), adjusted or not for age, gender and ethnic origin, and their corresponding 95\% confidence intervals (CI). The statistical package Epi Info was used to perform all these analyses.

The frequencies of the GSTM1, GSTT1 and combined GSTM1 and GSTT1 null genotypes were similar in patients and controls. Patients with the distinct genotypes of the GSTM1 and GSTT1 genes exhibited similar distribution to normal controls, suggesting that GST genotypes make no significant contribution to NEB, under the chemical exposures encountered in this study (Table 1).

In Brazil, workers are exposed predominantly to solvents, such as benzopyrene, hexachlorobenzene, ethylene oxide, dichloromethane and epoxybutanes, which are metabolised by the GSTM1 and GSTT1 enzymes (Ruiz et al., 1994; Queiroz et al., 1997; Hayes et al., 2005). The chemicals have been consistently associated with both AML and NEB in Brazil (Lorand et al., 1984; Ruiz et al., 1994; Queiroz et al., 1997; Augusto et al., 1999) and in other parts of the world (Cronkite et al., 1989; Ye and Song, 2005;

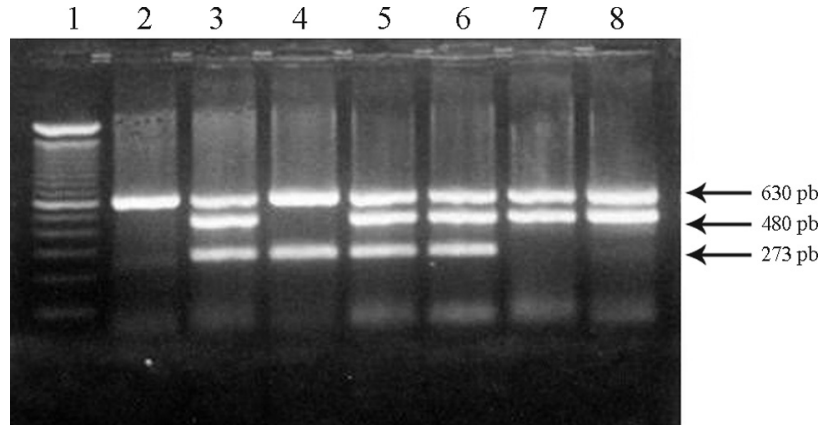

Figure 1 - Multiplex-PCR for detection of the wild and null alleles of the glutathione S-transferase mul (GSTM1) and thetal (GSTT1) genes. Ethidium bromide-stained $2 \%$ agarose gel showing products of $273 \mathrm{bp}$ and

Vaughan et al., 2005). These data supported the association of both GST null genotypes with increased risks for AML previously found by our group (Arruda et al., 2001). On this basis, the GST null genotypes were also expected to be associated with increased NEB risk. Surprisingly we found similar frequencies of the GST genotypes in our NEB patients and controls.

Unfortunately, there was no available data concerning the levels of benzene exposure of the NEB patients in this study and of the AML patients in our previous study (Arruda et al., 2001). We assumed similar exposures to benzene for patients in either group. Taking these results together, we hypothesise that different thresholds of chemical exposure relative to distinct GST genotypes may determine whether NEB or AML arises in chemical hazard exposed individuals from southeastern Brazil. Thus, those highly exposed to chemicals and homozygous for the null GST alleles may develop AML, since this seems to be more dependent on the GST pathway of carcinogen metabolism, whilst those individuals with less exposition to chemicals may be less dependent on carcinogen inactivation by the GST isoenzymes, and therefore more prone to present the benign form of occupational disease, NEB, without mediation of GST genotypes. Although indicative, these results must, however, be confirmed by prospective studies with

Table 1 - GSTM1 and GSTT1 genotypes in 55 patients with neutropenia due to exposure to benzene and 330 controls.

\begin{tabular}{|c|c|c|c|c|c|c|c|}
\hline & \multicolumn{2}{|c|}{ GSTM } & \multicolumn{2}{|c|}{ GSTT1 } & \multicolumn{3}{|c|}{ GSTM1/GSTT1 } \\
\hline & Present n $(\%)$ & Null n (\%) & Present n $(\%)$ & Null n (\%) & Both present $\mathrm{n}(\%)$ & One null n (\%) & Both null n (\%) \\
\hline Cases & $40(72.7)$ & $15(27.3)$ & $41(74.5)$ & $14(25.5)$ & $33(60.0)$ & $22(40.0)$ & $7(12.7)$ \\
\hline Controls & $202(61.2)$ & $128(38.8)$ & $265(80.3)$ & $65(19.7)$ & $159(48.2)$ & $149(45.1)$ & $22(6.7)$ \\
\hline OR (CI 95\%) & 1.0 (ref) & $\begin{array}{c}0.59 \\
(0.31-1.11)\end{array}$ & 1.0 (ref) & $\begin{array}{c}1.39 \\
(0.72-2.71)\end{array}$ & 1.0 (ref) & $\begin{array}{c}0.71 \\
(0.40-1.28)\end{array}$ & $\begin{array}{c}1.53 \\
(0.60-3.88)\end{array}$ \\
\hline Pvalue & \multicolumn{2}{|c|}{0.13} & \multicolumn{2}{|c|}{0.37} & & 0.30 & 0.43 \\
\hline OR* (CI 95\%) & 1.0 (ref) & $\begin{array}{c}0.61 \\
(0.31-1.21)\end{array}$ & 1.0 (ref) & $\begin{array}{c}1.54 \\
(0.75-3.14)\end{array}$ & 1.0 (ref) & $\begin{array}{c}0.74 \\
(0.39-1.38)\end{array}$ & $\begin{array}{c}0.82 \\
(0.65-4.81)\end{array}$ \\
\hline$P$ value & 0.16 & & 0.24 & & & 0.34 & 0.26 \\
\hline
\end{tabular}

n: number of cases; OR: odds ratio; *: adjusted OR by age, gender, and ethnic origin; CI: confidence interval. 
larger samples of NEB and AML patients and controls, with rigorous assessment of daily chemical exposures, and control of the influence of other polymorphic genes involved in benzene metabolism (Aydin-Sayitoglu et al., 2006).

\section{References}

Arruda VR, Lima CS, Grignoli CR, Melo MB, Lorand-Metze I, Alberto FL, Saad STO and Costa FF (2001) Increased risk for acute myeloid leukaemia in individuals with glutathione S-transferase mu 1 (GSTM1) and theta 1 (GSTT1) gene defects. Eur J Haematol 66:383-388.

Augusto LG, Fontbonne A, De Carvalho EM, Novaes EM and Novaes TC (1999) Socio-medical intervention in occupational health: Benzenism in Brazil. Int $\mathrm{J}$ Occup Environ Health 5:20-25.

Aydin-Sayitoglu M, Hatirnaz O, Erensoy N and Ozbek U (2006) Role of the CYP2D6, CYP1A1, CYP2E1, GSTT1, and GSTM1 genes in the susceptibility to acute leukemias. Am J Hematol 81:162-170.

Cronkite EP, Drew RT, Inoue T, Hirabayashi Y and Bullis JE (1989) Hematotoxicity and carcinogenicity of inhaled benzene. Environ Health Perspect 82:97-108.

Dale DC (2001) Neutropenia and Neutrophilia. In: Beutler E, Lichtman MA, Coller BS and Kipps TJ (eds) Hematology. Macgraw-Hill, New York, pp 823-834.

Hayes JD, Flanagan JU and Jowsey IR (2005) Glutathione transferases. Annu Rev Pharmacol Toxicol 45:51-88.
Lima CSP, Paula EV, Takahashi T, Saad STO, Lorand-Metze I and Costa FF (2006) Causes of incidental neutropenia in adult patients. Ann Hematol 85:705-709.

Lorand IGH, Souza CA and Costa FF (1984) Haematological toxicity associated with agricultural chemicals in Brazil. Lancet $1: 404$.

Queiroz MLS, Bincoleto C, Perlingeiro RCR, Souza CA and Toledo H (1997) Defective neutrophil function in workers occupationally exposed to hexachlorobenzene. Hum Exp Toxicol 16:322-326.

Ruiz MA, Augusto LGS, Vassallo J, Vigorito AC, Lorand-Metze I and Souza CA (1994) Bone marrow morphology in patients with neutropenia due to chronic exposure to organic solvents. Pathol Res Pract 190:151-154.

Vaughan AT, Betti CJ, Villalobos MJ, Premkumar K, Cline E, Jiang Q and Diaz MO (2005) Surviving apoptosis: A possible mechanism of benzene-induced leukemia. Chem Biol Interact 153:179-185.

Ye Z and Song H (2005) Glutathione S-transferase polymorphisms (GSTM1, GSTP1 and GSTT1) and the risk of acute leukaemia: A systematic review and meta-analysis. Eur J Cancer 41:980-989.

Watts RG (1999) Neutropenia. In: Lee GR, Foerster J, Lukens J, Paraskevas F, Greer JP, Rodgers GM and Wintrobe MM (eds) Wintrobe's Clinical Hematology. Williams and Wilkins, Baltimore, pp 1862-1888.

Associate Editor: Peter L. Pearson

License information: This is an open-access article distributed under the terms of the Creative Commons Attribution License, which permits unrestricted use, distribution, and reproduction in any medium, provided the original work is properly cited. 